\title{
Cerebral microbleeds in idiopathic normal pressure hydrocephalus
}

\author{
Elias Johansson ${ }^{*^{*}}$, Khalid Ambarki ${ }^{2,3}$, Richard Birgander ${ }^{2}$, Nazila Bahrami ${ }^{2}$, Anders Eklund ${ }^{2,3}$ and Jan Malm ${ }^{1}$
}

\begin{abstract}
Background: A vascular disease could be involved in pathophysiology of normal pressure hydrocephalus (INPH). If so, there should be an association between INPH and cerebral microbleeds (CMB). This study aims to analyze if CMB are associated with INPH.

Methods: In this case-control study we included 14 patients with INPH (mean age 76 years, $60 \%$ female) and 41 healthy controls (HeCo; mean age 71 years, $60 \%$ female). All were investigated with magnetic resonance imaging (MRI) using a T2*-sequence. The MRI exams were reviewed by two neuroradiologists for the presence of CMBs; the prevalence of findings of two or more CMBs was compared between INPH group and control group. After investigation, INPH patients underwent shunt surgery.
\end{abstract}

Results: Two or more CMB were detected more frequently in the INPH group compared to HeCo ( $n=6,43 \%$ vs. $\mathrm{n}=4,10 \% ; p=0.01$ ). Among the participants where MRI revealed $C M B$, the number of $C M B$ was higher among the INPH patients than the HeCo (median 8; IQR 2-34 vs. median 1; IQR 1-2; $p=0.005$ ).

Conclusions: This study supports a vascular component to the pathophysiology of INPH.

Keywords: Idiopathic normal pressure hydrocephalus, Magnetic resonance imaging, Cerebral microbleeds

\section{Background}

Idiopathic normal pressure hydrocephalus is a syndrome featuring communicating hydrocephalus in combination with a symptom triad of balance- and gait-disturbance, cognitive decline and urinary incontinence. In Sweden, the prevalence in the 70-79 year age group is $0.2 \%$, rising to nearly $6 \%$ in individuals over 80 years [1]. The pathophysiology is probably multi-factorial [2]. As INPH patients improve with cerebrospinal fluid (CSF) drainage, a CSF dynamic disturbance is usually considered the main etiological component. In recent years, small vessel disease has emerged as an alternative mechanism to explain part of the pathophysiological process in that vascular risk factors, manifest vascular disease, and white matter hyperintensities have all been associated with INPH [3].

\footnotetext{
*Correspondence: elias.johansson@umu.se

${ }^{1}$ Department of Pharmacology and Clinical Neuroscience, Norrlands

Universitetssjukhus, Umeå University, S-901 85 Umeå, Sweden

Full list of author information is available at the end of the article
}

Cerebral microbleeds are iron deposits in the brain that are associated pathologically with hemosiderinladen macrophages [4]. Mainly located around small vessels, and revealed in iron-sensitive MRI sequences, $\mathrm{CMB}$ are associated with moderate cognitive decline, several causes of dementia and several vascular risk factors including age, hypertension, lacunar infarctions, white matter lesions and diabetes [4-9]. However, the association between CMB and INPH has not been studied. The vascular hypothesis that part of the INPH syndrome could be explained by small vessel disease, would be strengthened if there is an association between INPH and $\mathrm{CMB}$. The aim of this study was to analyze if $\mathrm{CMB}$ are associated with INPH.

\section{Methods}

This was a retrospective case-control study. From a consecutive cohort of patients aged $>40$ years that underwent evaluation for ventriculomegaly, we included all $(\mathrm{n}=14)$ patients that: (1) were classified as probable $(n=9)$ or possible $(n=5)$ INPH according to guidelines 
[10] (2) underwent shunt surgery and (3) were examined with MRI including a T2*-sequence preoperatively. The included patients were evaluated between January 2008 and March 2009. Mean Evans index was 0.40 (range $0.36-0.49$ ). Mean symptom duration was 3.2 years (range 1-7). All INPH patients had gait disturbance (100\%), 13 (93\%) had incontinence and six (43\%) a mild cognitive impairment (defined as Mini Mental Examination score of 22-26). Cognitive status and number of vascular risk factors were not exclusion criteria for the INPH-patients as they were for the controls. All patients were treated with a ventriculo-peritoneal shunt. Postoperative improvement was defined as reduced need of walking aids and/or walking speed increased by $>15 \%$, assessed by comparison of pre-operative and postoperative standardized exams of gait and walking speed performed by experienced physiotherapists at our neurology ward. The mean delay between surgery and postoperative assessment was 17 weeks (range 13-24 weeks). Post-operative follow-up was available in 13 patients. Of these 13 patients, ten (77\%) improved, one ( $8 \%)$ did not improve despite that the shunt was patent. Two (15\%) patients died from perioperative complications within one month of the surgery.

Forty-one healthy controls ( $\mathrm{HeCo}$ ), who considered themselves healthy, were recruited between September 2007 and August 2008 through an advertisement in the local newspaper. HeCo were eligible if they did not have any psychiatric or neurologic disorder or signs of manifest atherosclerotic disease (such as stroke or myocardial infarction). Mini-mental state estimation $\geq 28$ points was required, without any contraindication for MRI examination. Not more than two vascular risk factors out of smoking, hypertension and hyperlipidemia were accepted, potential controls with three vascular risk factors were not enrolled in the study.

All subjects (patients and $\mathrm{HeCo}$ ) were examined with MRI using a hydrocephalus protocol with a Philips Achieva 3 Tesla machine and an 8-channel head coil. Beside standard T1, T2 and FLAIR sequences, the CMB were assessed using a $\mathrm{T} 2 \%$-weighted sequence (axial T2 FFE, $5 \mathrm{~mm}$ slice thickness and $1 \mathrm{~mm}$ intersection gap $(\mathrm{FOV}=230 \times 230 \mathrm{~mm}$, matrix acquisition $512 \times 512$, $\mathrm{TE} / \mathrm{TR}=833 \mathrm{~ms} / 16 \mathrm{~ms}$ ). The occurrences of CMB were independently evaluated by two senior neuroradiologists (NB, RB) using the T2* images. The other MRI data were used to rule out CMB-mimics and disagreements were resolved by consensus decision. The number of microbleeds was assessed using the Microbleed Anatomic Rating Scale (MARS) [11].

$\mathrm{CMB}$ were analyzed using median, intra-quartile range (IQR). Mann-Whitney test was used to compared INPH and controls. Cohen's Kappa values were calculated for inter-observer agreements. Where appropriate, we used $95 \% \mathrm{CI}, \chi^{2}-2$-test and $t$ test. A $p$ value of $<0.05$ was preselected as the threshold for statistical significance. IBM SPSS v20.0 software was used for all statistical analyses.

The study was approved by the regional ethical review board at Umeå University.

\section{Results}

This study included 55 subjects; 41 healthy controls and 14 INPH patients. See Table 1 for baseline comparisons. Altogether, 249 CMBs were observed in all subjects. Among patients with INPH, the median number of $\mathrm{CMB}$ was 0.5 (IQR 0-8), and among HeCo the median number of $C M B$ was 0 (IQR $0-1 ; p=0.035$ ). When only subjects with $\mathrm{CMB}$ were taken into account, the median number CMB was eight (IQR 2-34) in INPH and one (IQR 1-2) among the controls $(p=0.005)$.

The frequency of $\geq 2 \mathrm{CMB}$ was $43 \%$ in INPH and $10 \%$ in HeCo (Table $2 ; p=0.01$ ). However, frequency of $\geq 1$ CMB did not differ between the two groups significantly, as it was 50 and $29 \%$ in the INPH group and control group, respectively $(p=0.20)$. The distribution of $C M B$ is presented in Table 3.

A concerning finding was that the two INPH patients that died within 30 days of shunt placement surgery were the two patients with the highest number of CMB (34 and $174 \mathrm{CMB}$ ) in the study. The patient with $174 \mathrm{CMB}$ suffered an intracerebral hemorrhage and the other

Table 1 Baseline comparison between patients with idiopathic normal pressure hydrocephalus and healthy controls

\begin{tabular}{|c|c|c|c|}
\hline & $\begin{array}{l}\text { INPH } \\
(n=14)\end{array}$ & $\begin{array}{l}\text { Healthy controls } \\
(n=41)\end{array}$ & $P$ value \\
\hline Age mean (SD) & $76.4(5.1)$ & $70.5(5.4)$ & $0.001^{*}$ \\
\hline Women n (\%) & $6(43)$ & $18(44)$ & $1.0^{\mathrm{a}}$ \\
\hline Previous MI n (\%) & $0(0)$ & $0(0)$ & NA \\
\hline Previous stroke n (\%) & $0(0)$ & $0(0)$ & NA \\
\hline Current smoking n (\%) & $0(0)$ & $2(5)$ & $1.0^{\mathrm{a}}$ \\
\hline Hypertension ${ }^{b}$ n (\%) & $12(86)$ & $28(68)$ & $0.30^{\mathrm{a}}$ \\
\hline Treated hyperlipidemia n (\%) & $0(0)$ & $6(15)$ & $0.18^{\mathrm{a}}$ \\
\hline $0-2$ risk factors ${ }^{c}$ & $14(100)$ & $41(100)$ & NA \\
\hline 3 risk factors ${ }^{c}$ & $0(0)$ & $0(0)$ & \\
\hline $\begin{array}{l}\text { Anti-platelet or anti-coagu- } \\
\text { lant medication }\end{array}$ & $4(29)$ & $0(0)$ & $0.003^{\mathrm{a}}$ \\
\hline MMT mean (range) & $26.6(22-30)$ & $29.3(28-30)$ & $0.002^{*}$ \\
\hline
\end{tabular}

INPH idiopathic normal pressure hydrocephalus, $M I$ myocardial infarction, $M M T$ mini mental test, $S D$ standard deviation

* 2-sided t-test

a 2 -sided $x^{2}$-test

b Defined as either blood pressure $>140 / 90$ or current use of blood pressure lowering medication

c Refers to current smoking, hypertension and treated hyperlipidemia 
Table 2 Number of participants with cerebral microbleeds among idiopathic normal pressure hydrocephalus patients and healthy controls

\begin{tabular}{lll}
\hline & INPH & Healthy controls \\
\hline Number of subjects & 14 & 41 \\
0 CMB n (\%) & $7(50)$ & $29(71)$ \\
1 CMB n (\%) & $1(7)$ & $8(20)$ \\
$\geq 2$ CMB n (\%) & $6(43)$ & $4(10)$ \\
$\geq 5$ CMB n (\%) & $4(29)$ & $0(0)$ \\
\hline
\end{tabular}

Table 3 Location of the cerebral microbleeds among the patients with idiopathic normal pressure hydrocephalus and healthy controls

\begin{tabular}{lll}
\hline & INPH & Healthy controls \\
\hline Number of subjects with a CMB & 7 & 12 \\
Infratentorial, $\mathrm{n}(\%)$ & $4(57)$ & $2(17)$ \\
Subcortical ("deep"), $\mathrm{n}(\%)$ & $5(71)$ & $6(50)$ \\
Lobar, $\mathrm{n}(\%)$ & $4(57)$ & $4(57)$ \\
\hline
\end{tabular}

patient had a sepsis with intrathecal bacteremia. Agreement between the observers for the presence of $\geq 2 \mathrm{CMB}$ was $96 \%$ (53/55) with a kappa value of 0.89 (95\% CI 0.73-1.0), agreement between the observers for the presence/absence of any CMB was $98 \%(54 / 55)$ with a kappa value of 0.96 (95\% CI 0.88-1.0).

\section{Discussion}

This was a preliminary study, which revealed that the prevalence of CMBs was higher in patients with INPH than in healthy controls. Two serious adverse events occurred after shunt surgery, and these two patients had extensive CMB. There is no obvious causative link between CMB and the features of INPH such as large ventricles and periventricular edema [10]. The association is likely due to the fact that both CMB and INPH are associated with small vessel disease and vascular risk factors $[4-7,12]$. Hence CMB are more common in patients with INPH. A similar association with vascular disease has also been proposed for $\mathrm{CMB}$ and dementia [9], and for $\mathrm{CMB}$ and increased risk of cerebral hemorrhage in patients treated with intravenous thrombolysis [13].

Our estimation of the prevalence of any CMB (50\%) was similar to that of patients with ischemic stroke (49\%) and other vascular disorders such as vascular dementia (59 \%) and vascular Parkinsonism (56 \%); whereas similar non-vascular degenerative disorders have lower prevalence: Non-vascular dementia $21 \%$ and idiopathic Parkinsons disease $18 \%$ [9, 14, 15]. Thus, our findings contribute to a better understanding of the etiology of INPH and support the suggested association between INPH, small vessel disease and vascular risk factors $[6,7]$. Combined, these findings suggest that INPH is a combination of vascular disease and cerebrospinal fluid dynamic disorder. However, more detailed studies of the association between INPH and vascular risk factors are warranted. The two patients with the highest number of CMB died within 30 days after shunt placement. One of the causes of death (intracerebral hematoma) could reasonably be associated with CMB. It is well known that intracerebral hemorrhage can occur after severe complication to shunt placement [16]. As this is a small study, this might very well be a chance finding.

We used $\geq 2 \mathrm{CMB}$ as outcome in this study. We did not use the presence of any $(\geq 1) C M B$ as outcome as it is reasonable to argue that one $\mathrm{CMB}$ can be considered a normal variant rather than pathological: Although no $\mathrm{CMB}$ is most common among the elderly (85\%), the presence of any one $\mathrm{CMB}$ is also common ( $9 \%$ ); whereas only $6 \%$ has $\geq 2 \mathrm{CMB}[5,6]$. Also, $\geq 2 \mathrm{CMB}$ is required to cause a clinical effect in diseases [13, 17]. A considerable weakness of this study is the small sample size. Another weakness of the study was that the mean age of the INPH patients was higher than for HeCo because the controls were not recruited to match specific INPH patients. Since CMB prevalence increases with age, a part of the difference in CMB prevalence might have been caused by difference in age. However, in support of this study,agreement between the reviewers (kappa 0.89) was in the upper tier compared to previous studies using $\mathrm{T} 2$ * (kappa 0.33-0.88) [4].

\section{Conclusion}

Almost half of patients with INPH have several $(\geq 2)$ $\mathrm{CMB}$ and several $\mathrm{CMB}$ seems to be more common among patients with INPH than among controls. If these preliminary data can be confirmed, it would support the hypothesis that INPH pathophysiology includes a vascular component.

\section{Abbreviations}

CMB: cerebral microbleeds; CSF: cerebrospinal fluid; $\mathrm{HeCo}$ : healthy controls; INPH: idiopathic normal pressure hydrocephalus; IQR: inter quartile range; SD: standard deviation

\section{Authors' contributions}

EJ Co-designed the study, analyzed the material, wrote the first draft of the manuscript, KA Co-designed the study, gave critical comments to the manuscript, RB Co-designed the study, reviewed images for CMB, gave critical comments to the manuscript, NB Reviewed images for $\mathrm{CMB}$, gave critical comments to the manuscript, AE Co-designed the study, gave critical comments to the manuscript, JM Main study designer, gave critical comments to the manuscript. All authors read and approved the final manuscript. 


\section{Author details}

${ }^{1}$ Department of Pharmacology and Clinical Neuroscience, Norrlands Universitetssjukhus, Umeå University, S-901 85 Umeå, Sweden. ${ }^{2}$ Department of Radiation Sciences, Umeå University, Umeå, Sweden. ${ }^{3}$ Centre for Biomedical Engineering and Physics, Umeå University, Umeå, Sweden.

\section{Acknowledgements}

The authors want to acknowledge the assistance of research nurse Kristin Nyman in the data collection. This project was financed by the Swedish Research Council (Grant 621-2011-5216).

\section{Competing interests}

The authors declare that they have no competing interests.

Received: 9 November 2015 Accepted: 3 February 2016 Published online: 10 February 2016

\section{References}

1. Jaraj D, Rabiei K, Marlow T, et al. Prevalence of idiopathic normal-pressure hydrocephalus. Neurology. 2014;82:1449-54.

2. Malm J, Eklund A. Idiopathic normal pressure hydrocephalus. Pract Neurol. 2006:6:14-27.

3. Malm J, Graff-Radford NR, Ishikawa M, et al. Influence of comorbidities in idiopathic normal pressure hydrocephalus — research and clinical care: a report of the ISHCSF task force on comorbidities in INPH. Fluids Barriers CNS. 2013;10:22.

4. Greenberg SM, Vernooij MW, Cordonnier C, et al. Microbleed study group. cerebral microbleeds: a guide to detection and interpretation. Lancet Neurol. 2009;8:165-74.

5. Sveinbjornsdottir S, Sigurdsson S, Aspelund T, et al. Cerebral microbleeds in the population based AGES Reykjavik study: prevalence and location. J Neurol Neurosurg Psychiatry. 2008;79:1002-6.
6. Vernooij MW, van der Lugt A, Ikram MA, et al. Prevalence and risk factors of cerebral microbleeds: the Rotterdam scan study. Neurology. 2008;70:1208-14.

7. Roob G, Schmidt R, Kapeller P, et al. MRI evidence of past cerebral microbleeds in a healthy elderly population. Neurology. 1999;52:991-4.

8. Poels MM, Ikram MA, van der Lugt A, et al. Cerebral microbleeds are associated with worse cognitive function: the Rotterdam scan study. Neurology. 2012;78:326-33.

9. Shams S, Martola J, Granberg T, et al. Cerebral Microbleeds: different Prevalence, topography, and risk factors depending on dementia diagnosisthe Karolinska imaging dementia study. Am J Neuroradiol. 2015;36:661-6.

10. Relkin N, Marmarou A, Klinge P, et al. Diagnosing idiopathic normalpressure hydrocephalus. Neurosurgery. 2005:57(3 suppl):S4-16.

11. Gregoire SM, Chaudhary UJ, Brown MM, et al. The microbleed anatomical rating scale (MARS): reliability of a tool to map brain microbleeds. Neurology. 2009;73:1759-66.

12. Krauss JK, Regel JP, Vach W, et al. Vascular risk factors and arteriosclerotic disease in idiopathic normal-pressure hydrocephalus of the elderly. Stroke. 1996;27:24-9.

13. Dannenberg S, Scheitz JF, Rozanski M, et al. Number of cerebral microbleeds and risk of intracerebral hemorrhage after intravenous thrombolysis. Stroke. 2014;45:2900-5.

14. Kim JH, Park J, Kim YH, Ma HI, Kim YJ. Characterization of cerebral microbleeds in idiopathic parkinson's Disease. Eur J Neurol. 2015:22:377-83.

15. Yamada S, Saiki M, Satow T, et al. Periventricular and deep white matter leukoaraiosis have a closer association with cerebral microbleeds than age. Eur J Neurol. 2012;19:98-104.

16. Misaki K, Uchiyama N, Hayashi Y, Hamada J. Intracerebral hemorrhage secondary to ventriculoperitoneal shunt insertion-four case reports. Neurol Med Chir (Tokyo). 2010;50:76-9

17. Knudsen KA, Rosand J, Karluk D, et al. Clinical diagnosis of cerebra amyloid angiopathy: validation of the Boston Criteria. Neurology. 2001;56:537-9.

\section{Submit your next manuscript to BioMed Central and we will help you at every step:}

- We accept pre-submission inquiries

- Our selector tool helps you to find the most relevant journal

- We provide round the clock customer support

- Convenient online submission

- Thorough peer review

- Inclusion in PubMed and all major indexing services

- Maximum visibility for your research

Submit your manuscript at www.biomedcentral.com/submit

\section{() BioMed Central}

